\title{
Skin hydration is significantly increased by a cream formulated to mimic the skin's own natural moisturizing systems
}

This article was published in the following Dove Press journal: Clinical, Cosmetic and Investigational Dermatology

\section{Fabrizio Spada Tanya M Barnes Kerryn A Greive Research and Development, Ego Pharmaceuticals Pty Ltd, Braeside, VIC, Australia}

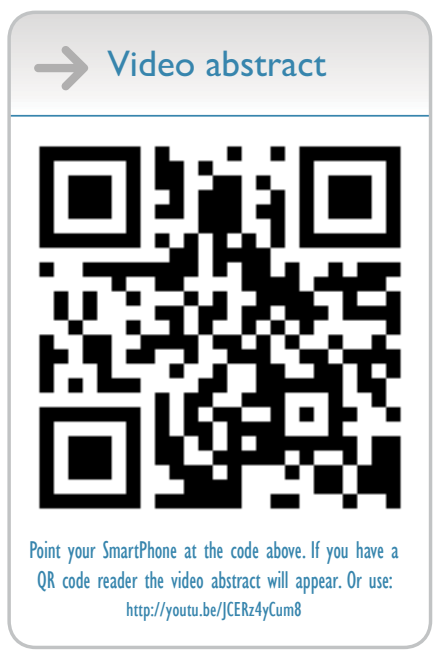

Correspondence: Fabrizio Spada Ego Pharmaceuticals Pty Ltd, 2I-3I Malcolm Road, Braeside, VIC 3195, Australia

Tel +6I 395868874

Fax +6I 395807647

Email fabrizio.spada@egopharm.com
Background: Moisturizers are topical products designed to improve and maintain the skin barrier function and to help prevent dry skin.

Materials and methods: A new moisturizer (Ceramide cream) was formulated containing ingredients which mimic the skin's own natural moisturizing systems. Corneometry was performed at baseline, 2, 4, 6 and 24 hours following a single application of Ceramide cream to healthy skin, and compared to three reference moisturizers available over-the-counter, and placebo. Transepidermal water loss (TEWL) was also measured following a single application of Ceramide cream compared to baseline, and its safety was assessed by repeat insult patch test, ophthalmologist and pediatric testing.

Results: A single topical application of either the Ceramide cream or the three reference moisturizers resulted in a significant increase in skin hydration over time $(P<0.001)$. The placebo cream did not significantly increase skin hydration at any time point. At 24 hours post-application, skin hydration measured for Ceramide cream was significantly greater $(P<0.05)$ than that measured for all three of the reference moisturizers tested. Ceramide cream was also found to significantly decrease TEWL $(P<0.001)$ over 24 hours, and was shown to be non-sensitizing to the skin of both adults and children and non-irritating to the skin, eyes and related eye area.

Conclusion: Ceramide cream increases skin hydration and improves barrier function which may make it suitable for use on dry skin.

Keywords: ceramide, moisturizer, stratum corneum, dry skin, humectant, emollient, occludent, transepidermal water loss, natural moisturizing factor

\section{Introduction}

A properly functioning stratum corneum (SC) is essential for healthy skin. ${ }^{1}$ To maintain integrity, the SC employs a number of natural systems to keep water in or on the skin. ${ }^{2}$ One of these systems is the natural moisturizing factor (NMF) which is composed predominately of free amino acids, pyrrolidone carboxylic acid (PCA), urocanic acid, lactic acid and urea. ${ }^{3}$ The NMF components are highly efficient humectants that attract and bind water from the atmosphere, drawing it into the corneocytes. ${ }^{4}$ The structure of the SC is arranged in a 'brick and mortar' mosaic of flattened corneocytes ('bricks'), embedded in a lipid-enriched extracellular matrix ('mortar'), organized into parallel stacks of lamellar bilayers. ${ }^{2}$ These water-repellent lamellar bilayers are composed of a blend of ceramides, free fatty acids and cholesterol and restrict not only the outward flow of water and electrolytes, but the inward absorption of chemical substances, allergens and microbial pathogens. ${ }^{5}$ Further, sebum is produced by the sebaceous glands and forms a film over the top of the skin. ${ }^{6}$ 
Dry skin or xeroderma is an extremely common problem which can be induced by complex interactions between environmental and individual factors including, but not limited to: low environmental temperature, low humidity, exposure to chemicals, microorganisms, aging, psychological stress, atopic dermatitis and eczema. ${ }^{7}$ Moisturizers are topical products designed to improve and maintain the skin barrier function and to help prevent dry skin. It is common to think that a moisturizer adds water to the skin; however, this is a misunderstanding. Rather, a moisturizer works by preventing or reducing water evaporation from the skin. ${ }^{8}$ This action allows the skin to rehydrate from within. There are three classes of chemical ingredients that regularly serve as moisturizers: occlusives, humectants and emollients. ${ }^{79}$ Often these chemicals are either the same as or similar to natural components in the SC. They are often used in combination, with some ingredients providing overlap of characteristics. ${ }^{9}$

Occlusive agents serve to reduce transepidermal water loss (TEWL) by forming a hydrophobic barrier film over the skin surface to prevent evaporation of water from the SC, trapping water in the skin's uppermost layers. ${ }^{10}$ Humectants are hygroscopic substances which attract water and moisture. ${ }^{10}$ When humectants are present on the skin, water from the dermis is absorbed into the epidermis. Minimal water is absorbed from the environment. ${ }^{11}$ Many of these chemicals are the same molecules that form the NMF. The third class, emollients, are chemicals that improve the "feel" of the skin by filling the spaces in between corneocytes and also provide what has been termed "skin slip" or lubricity, imparting a sense of softness and plasticity. ${ }^{12}$

The newest generation of moisturizers also contain barrier repair ingredients in addition to traditional moisturizer components. The most common of such ingredients are ceramides, free fatty acids and cholesterol which help replace the deficient lipids in some skin diseases characterized by barrier impairment, such as eczema and psoriasis. ${ }^{13}$

There are a vast array of moisturizers available in the market today and consumer demand for these products is growing. A US study found that moisturizers are the third most commonly recommended over-the-counter (OTC) topical skin product (13.4\%) after hydrocortisone (27.6\%) and anti-infectives (23.4\%). ${ }^{14}$

The aim of this pilot study was to assess the effect of a newly formulated moisturizer (referred to as Ceramide cream) on skin hydration as measured by corneometry in individuals with normal skin compared to three reference OTC moisturizers available in Australia. Ceramide cream contains carefully selected ingredients designed to mimic the skin's own natural systems to enhance and maintain skin barrier integrity and prevent dry skin. The effect of Ceramide cream on TEWL was also measured by tewameter and its safety was assessed by repeat insult patch test (RIPT), ophthalmologist and pediatric testing. These studies will determine whether Ceramide cream should be tested more extensively and for a longer time period in clinical trials in patients with diseases characterized by skin barrier impairment, such as eczema.

\section{Materials and methods Topical preparations}

A moisturizer, QV Intensive with Ceramides - Light Moisturizing Cream (referred to as Ceramide cream), was formulated and tested containing a combination of carefully selected ingredients as shown in Table 1. This moisturizer was designed to mimic the skin's own natural systems to enhance and maintain skin barrier integrity (Ego Pharmaceuticals Pty Ltd, Braeside, VIC, Australia). A placebo cream was also formulated which did not include the skin active ingredients.

Topical moisturizers used as reference products in this study which are available OTC include: Dermeze ${ }^{\circledR}$ Thick Cream (referred to as Reference Product 1; Aspen Pharmacare Australia Pty Ltd, St Leonards, NSW, Australia), Physiogel ${ }^{\circledR}$ AI Cream (referred to as Reference Product 2; Glaxo Operations UK Ltd Trading as Glaxo Wellcome Operations, Durham, UK) and CeraVe ${ }^{\circledR}$ Moisturizing Lotion (referred to as Reference Product 3; Valeant Consumer Products, a Division of Valeant Pharmaceuticals, Bridgewater, NJ, USA). Ingredients present in these formulations are shown in Table 1. These moisturizers were chosen as they were amongst the most popular moisturizers used in Australia at the time of the study.

\section{Study participants}

The skin hydration and TEWL studies were approved by the Independent Ethics Committee of Dermatest Pty Ltd (Rockdale, NSW, Australia), the RIPT and ophthalmology studies were approved by the Institutional Review Board of Cantor Research Laboratories, Inc. (Blauvelt, NY, USA) and the pediatric studies were approved by the Institutional Review Board of Essex Testing Clinic, Inc. (Verona, NJ, USA). All operate in accordance with the International Conference on Harmonization Good Clinical Practice guidelines.

Eligible participants included healthy men and women aged between 18 and 70 years who: were not taking medication or under the care of a doctor for a period of 1 month prior to commencement and throughout the entire test period; completed an extensive medical history form; were free of any dermatological or systemic disorder that would interfere 
Table I Ingredients listed on the product labels of moisturizers tested in this study

\begin{tabular}{|c|c|c|c|c|c|}
\hline Ingredient class & Ceramide cream & Placebo cream & Reference product I & Reference product 2 & Reference product 3 \\
\hline Base & Water & Water & Water & Water & Water \\
\hline \multirow[t]{2}{*}{ Humectant } & Glycerin & & & Betaine & Glycerin \\
\hline & Sodium $\mathrm{PCA}^{\mathrm{a}}$ & & & Glycerin & \\
\hline \multirow[t]{2}{*}{ Occludent } & Dimethicone & & White soft paraffin & Squalane & Caprylic/capric triglyceride \\
\hline & Petroleum & & & & Dimethicone \\
\hline \multirow[t]{3}{*}{ Emollient } & Paraffinium liquidum & & Liquid paraffin & Elaeis guineensis oil & \\
\hline & I,2-hexanediol & & & Olea europaea fruit oil & \\
\hline & caprylyl glycol & & & Olus & \\
\hline \multirow[t]{2}{*}{ Ceramide promoter } & Niacinamide & & & & Phytosphingosine \\
\hline & Lactic acid ${ }^{a}$ & & & & \\
\hline \multirow{6}{*}{$\begin{array}{l}\text { Ceramide/ } \\
\text { cholesterol/fatty acid }\end{array}$} & Ceramide NP & & & & Ceramide I \\
\hline & & & & & \\
\hline & Ceramide EOP & & & & Ceramide 3 \\
\hline & Cholesterol & & & & Ceramide 6-II \\
\hline & Carthamus tinctorius & & & & Cholesterol \\
\hline & (safflower) seed oil & & & & \\
\hline \multirow[t]{12}{*}{ Other } & Cetearyl alcohol & Cetearyl alcohol & Cetearyl alcohol & Pentylene glycol & Behentrimonium methosulfate \\
\hline & Ceteareth-20 & Ceteareth-20 & Ceteareth-20 & Acetamide MEA & Carbomer \\
\hline & Glyceryl stearate SE & Glyceryl stearate SE & Citric acid & Carbomer & Ceteareth-20 \\
\hline & Laureth-3 & Laureth-3 & Methylparaben & Hydrogenated lecithin & Cetearyl alcohol \\
\hline & Sodium hydroxide & Methylparaben & Propylparaben & Hydroxyethylcellulose & Dipotassium EDTA \\
\hline & Stearic acid & Propylparaben & Trisodium citrate & Palmitamide MEA (PEA) & Hyaluronic acid \\
\hline & Xanthan gum & Stearic acid & & Sarcosine & Methylparaben \\
\hline & & Xanthan gum & & Sodium carbomer & Polyglyceryl-3 diisostearate \\
\hline & & & & Xanthan gum & Polysorbate 20 \\
\hline & & & & & Potassium phosphate \\
\hline & & & & & Propylparaben \\
\hline & & & & & Xanthan gum \\
\hline
\end{tabular}

Notes: aAlso an NMF component.

Abbreviations: PCA, pyrrolidone carboxylic acid; NMF, natural moisturizing factor.

with the results; were available for the study duration and gave written informed consent. Exclusion criteria were individuals: under doctor's care; taking medication which could mask or interfere with test results; with a history of sensitivity to cosmetics in general and moisturizers in particular; with any form of skin cancer or any disease that could interfere with test results; diagnosed with chronic skin allergies; with excessive hair on the test sites and females who indicated that they were pregnant or nursing an infant.

\section{Measurement of skin hydration}

In order to pre-condition the test sites and to keep topical treatments consistent, participants were required to abstain from using deodorant soaps, moisturizing soaps or cosmetic moisturizers on the test area for a period of 1 week prior to study commencement and during the course of the study. At the completion of the washout period, participants were randomized ( $\mathrm{n}=10$ for each moisturizer) to receive a single application of either Ceramide cream, Reference Products 1-3 or placebo cream applied liberally and evenly to the volar forearm through volumetric syringes in a double-blind manner. The opposing volar forearm served as the untreated control.

Before each set of measurements, participants were required to equilibrate in a closed environment with a constant temperature $\left(20^{\circ} \mathrm{C} \pm 2^{\circ} \mathrm{C}\right)$ and humidity $(45 \%-55 \%$ $\mathrm{RH})$. The relative water content in the SC, or skin hydration, was measured using a corneometer (Model CM 825 PC corneometer, Courage and Khazaka, Germany) at $t=0$ (pre-application) and at 2, 4, 6 and 24 hours post-application in triplicate, expressed in arbitrary units. ${ }^{15}$ Participants were required to remain in the laboratory for the first day of the study and then return as required. Any adverse events that were observed or reported during the entire study period were documented by the investigator.

\section{Measurement of TEWL}

The pre-conditioning of test sites was the same as that described for the measurement of skin hydration. A single 
application of Ceramide cream was applied liberally and evenly to the volar forearm of participants through volumetric syringes in a double-blind manner. The opposing volar forearm served as the untreated control.

Before each set of measurements, participants were required to equilibrate in a closed environment with a constant temperature $\left(20^{\circ} \mathrm{C} \pm 2^{\circ} \mathrm{C}\right)$ and humidity $(45 \%-55 \% \mathrm{RH})$. Measurement of TEWL was performed using a Tewameter (Model TM 210 Tewameter $^{\circledR}$, Courage + Khazaka, Germany) at $t=0$ (pre-application) and at 2, 4, 6 and 24 hours postapplication in triplicate, expressed in $\mathrm{g} / \mathrm{hm}^{2} .{ }^{16}$ Participants were required to remain in the laboratory for the first day of the study and then return as required. In addition, any adverse events that were observed or reported during the entire study period were documented by the investigator.

\section{RIPT}

Participants were requested to bathe or wash as usual before the test. A total of $0.2 \mathrm{~mL}$ of Ceramide cream was placed onto a semi-occlusive, hypoallergenic patch (Parke-Davis Hypoallergenic Readi-Bandage; $20 \times 20 \mathrm{~mm}$ Webril affixed to the center of a $40 \times 40 \mathrm{~mm}$ adhesive bandage). The patch was then affixed directly to the skin of the infrascapular regions of the back, to the right or left of the midline and the participant was allowed to return home with instructions not to wet or expose the test area to direct sunlight. After 24 hours the patch was removed by the participant at home. This procedure was repeated until a series of nine consecutive 24 hours exposures were made for every Monday, Wednesday and Friday for three consecutive weeks.

In the event of an adverse reaction, the area of erythema and edema was measured. Edema was estimated by the evaluation of the skin in respect to the contour of the unaffected normal skin. Reactions were scored just before applications two through nine and at the next test date following application nine. Subjects were then given a 10- to 14-day rest period after which a challenge or retest dose was applied once to a previously unexposed test site. The retest dose was equivalent to any one of the original nine exposures. Reactions were scored 24 and 48 hours after application. Scoring was performed using the following scale; 0 : no evidence of any effect, ?: barely perceptible, minimal faint (light pink) uniform or spotty erythema, 1 : mild pink uniform erythema covering most of the contact site, 2: moderate pink/red erythema visibly uniform in entire contact area, 3: marked bright red erythema with accompanying edema, petechiae or papules, 4: severe deep red erythema with vesiculation or weeping with or without edema.

\section{Ophthalmologist testing}

Additional inclusion criteria to that described in the study participants section above were individuals: free of any ocular disorder that would interfere with the results; wearing any variety of contact lens every day; had no subjective ocular irritation (stinging, burning, itching, dryness or foreign object sensation). Additional exclusion criteria were individuals: who were currently using ophthalmic drugs other than contact lens solutions or disinfectants, rewetting solutions and lubricants; with known allergies or skin and/or eye conditions which would interfere with the results; any known abnormal reaction to eye area products; who were currently using any topical eye products.

Participants were randomized and required to apply Ceramide cream to either the left or right eye area twice daily for 4 weeks. The participant's eyes and eye area, including eyelids, were examined by an ophthalmologist using an ophthalmoscope and scored at $t=0$ (pre-application), and following 1, 2, 3 and 4 weeks of Ceramide cream application, specifically looking for irritation, swelling, inflammation, discharge, erythema or any other abnormalities. Skin grading of the eye area was scored using the following scale; 0 : no reaction, 1+: weak (non-vesicular) showing erythema, infiltration and possible papules, $2+$ : strong reaction with vesicles in addition to erythema and infiltration and 3+: extreme reaction, bullous or ulcerative. ${ }^{17}$ Eye grading was scored using the following scale: 0 : no reaction, $1+$ : conjunctivae (palpebral and bulbar) injected above normal with possible chemosis (swelling) and no discharge, 2+: conjunctivae injected above normal, obvious swelling and possible discharge and 3+: conjunctivae more diffuse, crimson red, individual vessels not easily discernible, excessive swelling and/or discharge. ${ }^{18}$

\section{Pediatric testing}

Ceramide cream was tested according to a single-blind, homeuse, pediatrician-monitored protocol that was conducted with pediatric subjects aged 6-36 months who had sensitive skin. A parent or legal guardian provided written informed consent for the pediatric participants. Participants were randomized and required to apply Ceramide cream to either the left or right arm and leg at least once daily for 2 weeks. Comments were noted in a daily diary and parents/legal guardians were required to call the clinic to report on progress after 1 week. Children were examined by a pediatrician pre- and post-test.

\section{Statistical analyses}

Statistical analyses were conducted using SPSS version 25 (IBM, Armonk, NY, USA). To compare hydration scores 
across the five moisturizers tested, mean difference scores for the treated vs untreated conditions at each time point were calculated. A repeated measures ANOVA with a GreenhouseGeisser correction was performed and least significant difference post hoc analyses conducted.

The percentage change in TEWL was calculated by the following equation: Percentage change TEWL $=[(\mathrm{a}-\mathrm{b}) /$ b] $\times 100$, where $a=$ individual value of TEWL at each individual time point and $b=$ zero hour value of TEWL. The Student's $t$-test was used to compare the mean values and \% change in TEWL at each time point compared to pre-application. A statistical significance level of $P<0.05$ was applied to all analyses.

\section{Results}

\section{Skin hydration following a single application of moisturizers}

Fifty participants (12 male, 38 female) aged between 18 and 63 years ( $43.0 \pm 13.5$ years) were enrolled in this randomized, double-blind study. All participants completed the study and no adverse event was observed.

The mean difference in hydration scores (treated minus untreated) for each of the moisturizers tested over 24 hours are presented in Figure 1. There was no significant difference in hydration scores between any of the moisturizers at baseline. A single topical application of either the Ceramide cream or the three reference moisturizers resulted in a significant increase in skin hydration over time $(P<0.001)$. Topical application of the placebo cream did not significantly increase skin hydration at any time point. Ceramide cream showed

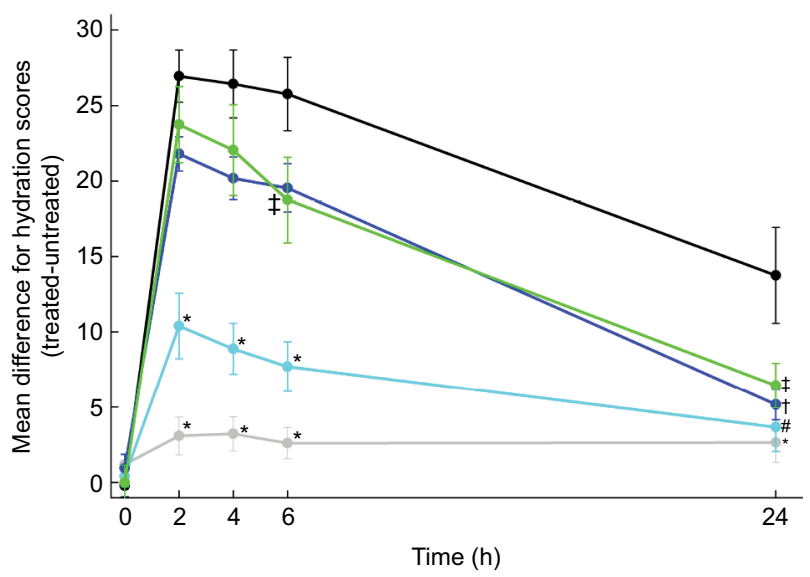

Figure I Mean difference for hydration scores (treated minus untreated) over time (h) following the single application of $(\bullet)$ Ceramide cream, ( $)$ placebo cream, ( $)$ Reference Product I, (•) Reference Product 2 and $(\bullet)$ Reference Product $3(\mathrm{n}=10$ for each group).

Notes: ${ }^{*} P<0.0001$ vs Ceramide cream, ${ }^{\#} P<0.001$ vs Ceramide cream, ${ }^{\wedge} P<0.01$ vs Ceramide cream and $¥ P<0.05$ vs Ceramide cream. the greatest increase in hydration compared to the bare skin control at 2 through to 24 hours post-application compared to the three reference moisturizers and placebo (Figure 1). Conversely, the placebo cream and Reference Product 1 had the lowest increase in hydration, with differences between these two products only statistically significant at 2 hours post-application $(P=0.017)$. At 24 hours post-application, Ceramide cream continued to have a significantly greater increase in hydration compared to Reference Product 1 $(P=0.001)$, Reference Product $2(P=0.003)$ and Reference Product $3(P=0.012)$.

\section{TEWL following a single application of Ceramide cream}

Ten participants (two male, eight female) aged between 21 and 61 years $(47.7 \pm 3.9$ years) were enrolled in the study. All participants completed the study and no adverse event was observed. Table 2 shows the mean TEWL measurements (g/ $\mathrm{hm}^{2}$ ) as well as the mean percentage decrease in TEWL following a single topical application of Ceramide cream over time. The mean baseline TEWL was $8.45 \pm 0.65 \mathrm{~g} / \mathrm{hm}^{2}$. Two hours post-application, TEWL significantly decreased by $\sim 25 \%$ compared to baseline. Four hours post-application, TEWL was still significantly decreased by $\sim 22 \%$ compared to baseline, which was maintained up to 24 hours post-application $(P<0.001$ for all time points).

\section{RIPT}

A total of 103 participants (19 male, 84 female) aged between 21 and 70 years were enrolled in the study. Two participants were discontinued from the study due to their absence on moisturizer application days. The RIPT showed that none of the participants had an adverse reaction of any kind to Ceramide cream when tested neat under semi-occlusion, and therefore Ceramide cream may be considered as nonirritating and non-sensitizing to the skin.

Table 2 TEWL measurements $\left(\mathrm{g} / \mathrm{hm}^{2}\right)$ following a single topical application of Ceramide cream over time $(\mathrm{h})$. The mean percentage change in TEWL compared to baseline $(t=0)$ is given in parentheses.

\begin{tabular}{ll}
\hline Time (h) & TEWL (g/hm $\mathbf{h m}^{2}$ \\
\hline 0 & $8.45 \pm 0.65$ \\
2 & $6.35 \pm 0.56(-25.4 \pm 1.2)^{*}$ \\
4 & $6.56 \pm 0.44(-21.6 \pm 2.2)^{*}$ \\
6 & $6.58 \pm 0.40(-21.0 \pm 2.6)^{*}$ \\
24 & $6.54 \pm 0.38(-21.2 \pm 3.0)^{*}$ \\
\hline
\end{tabular}

Notes: ${ }^{*} P<0.001$ vs $t=0$. Results are presented as mean \pm SEM. Abbreviation: TEWL, transepidermal water loss. 


\section{Ophthalmologist testing}

Fifty-two participants (four male, 48 female) aged between 22 and 60 years $(41.3 \pm 1.5$ years $)$ were enrolled and completed the study, including 26 non-contact lens wearers and 26 contact lens wearers. None of the participants had an adverse reaction of any kind to the Ceramide cream over the 4-week test period. Therefore, when tested neat under exaggerated conditions, Ceramide cream may be considered as non-irritating to the eyes and related eye area.

\section{Pediatric testing}

Sixteen boys and 15 girls aged from 6 to 36 months $(21.7 \pm 1.6$ months) were enrolled in the study, with six children in each age group: 6-12 months, 13-18 months, 19-24 months, 25-30 months and 31-36 months. One child was discontinued from the study for reasons unrelated to the study protocol. No test-related irritation was observed by the pediatrician and no safety-related comments were made by any participants or their parents or guardians at any time during the course of the study.

\section{Discussion}

Moisturizing treatment involves a four-step process: 1) repairing the skin barrier, 2) increasing water content, 3) reducing TEWL and 4) restoring the lipid barriers' ability to attract, hold and redistribute water. ${ }^{19,20}$ In addition, an ideal moisturizer should be cosmetically elegant and acceptable, moisturizing to sensitive skin (hypoallergenic, non-sensitizing, fragrance free and non-comedogenic), offered at an affordable price, long lasting and absorbed rapidly providing immediate hydration. ${ }^{19,20}$

All moisturizers tested in this study were shown to significantly increase skin hydration for up to 24 hours. At 24 hours post-application, skin hydration measured for Ceramide cream was found to be significantly greater compared to that measured for the reference moisturizers. Ceramide cream was also found to significantly decrease TEWL for up to 24 hours, was non-sensitizing to the skin of both adults and children and non-irritating to the skin, eyes and related eye area.

The effect of Ceramide cream on enhancing skin barrier function and hydration might be explained by its unique ingredients. Specifically, PCA which is a filaggrin breakdown product and part of the skin's NMF is present as sodium PCA in Ceramide cream, the form of PCA most used in topical preparations, which helps to restore the hydration of the SC. ${ }^{3}$ Lactic acid also forms part of the NMF, and together with nicotinamide have been shown to promote ceramide biosynthesis and thus strengthen the skin barrier. ${ }^{21,22}$ Ceramide cream also contains ceramides 1 and 3 , cholesterol and linoleic acid from safflower oil in a 3:1:1 molar ratio as it has been shown that these ingredients must be delivered in the correct ratio to have a positive effect on the integrity of the skin barrier. ${ }^{4}$

In addition to these novel ingredients, Ceramide cream contains humectants which act similarly to NMF in corneocytes, an occludent which simulates sebum and natural lipids found on the skin, and emollients which act like the intracellualar bilayers of the SC (Table 1). ${ }^{7}$ The ingredients in this "triple moisturizing system" are combined in a specific ratio to mimic the natural $\mathrm{SC}$ components as it has been shown that each of these components are important to maintain healthy skin barrier function. ${ }^{1,2}$ Ceramide cream is also fragrance and preservative free as these ingredients are known to be some of the most common allergens. ${ }^{23}$ Furthermore, Ceramide cream is formulated with an acidic $\mathrm{pH}$ which has been shown to be optimal for healthy skin. ${ }^{24}$

The level of ceramides has been shown to be greatly reduced in the SC of patients with atopic dermatitis. It has subsequently been concluded that an insufficiency of ceramides in the SC is an important factor in atopic dry skin. ${ }^{2,25}$ As a result of this finding, ceramides have been added to many moisturizers used in the treatment of both atopic and normal skin. Further clinical studies are underway to determine longer term effects of Ceramide cream on skin hydration, signs of eczema and patient quality of life in patients with eczema.

A limitation of this study is that a lotion (Reference Product 3) was included in the panel of reference products tested and compared to a cream. This choice was made on the basis that this product was a popular moisturizer available in Australia at the time of the study. A further limitation is that the effect of the reference products on TEWL was not determined, however, given the hydration results all other parameters were studied on ceramide cream alone to understand the properties of this newly formulated cream.

In conclusion, topical application of the Ceramide cream moisturizer leads to increased skin hydration and decreased TEWL making it suitable to help restore xerotic skin. Maintenance of skin barrier function is vital to mitigate the skin's susceptibility to irritants, allergens, and microbes.

\section{Acknowledgments}

The authors would like to thank John Staton, Dermatest Pty. Ltd (Rockdale, NSW, Australia), for assistance in performing these studies. Ego Pharmaceuticals Pty Ltd sponsored this study and is the manufacturer of Ceramide cream. 


\section{Disclosure}

FS, TMB and KAG are employed by Ego Pharmaceuticals, Pty Ltd. The authors report no other conflicts of interest in this work.

\section{References}

1. Del Rosso JQ, Levin J. The clinical relevance of maintaining the functional integrity of the stratum corneum in both healthy and diseaseaffected skin. J Clin Aesthet Dermatol. 2011;4(9):22-42.

2. Harding CR. The stratum corneum: structure and function in health and disease. Dermatol Ther. 2004;17(Suppl 1):6-15.

3. Fowler J. Understanding the role of natural moisturizing factor in skin hydration. Prac Dermatol. 2012; July.

4. Rawlings AV, Scott IR, Harding CR, Bowser PA. Stratum corneum moisturization at the molecular level. J Invest Dermatol. 1994;103(5):731-741.

5. Saji D, Asiniwasis R, Skotnicki-Grant S. A look at epidermal barrier function in atopic dermatitis: physiologic lipid replacement and the role of ceramides. Skin Therapy Lett. 2012;17(7):6-9.

6. Boer M, Duchnik E, Maleszka R, Marchlewicz M. Structural and biophysical characteristics of human skin in maintaining proper epidermal barrier function. Postepy Dermatol Alergol. 2016;33(1):1-5.

7. Sethi A, Kaur T, Malhotra SK, Gambhir ML. Moisturizers: the slippery road. Indian J Dermatol. 2016;61(3):279-287.

8. Greive K. Cleansers and moisturisers: The basics. Wound Pract Res. 2015;23(2):76-81.

9. Rawlings AV, Canestrari DA, Dobkowski B. Moisturizer technology versus clinical performance. Dermatol Ther. 2004;17(Suppl 1):49-56.

10. Levin J, Miller R. A guide to the ingredients and potential benefits of over-the-counter cleansers and moisturizers for rosacea patients. J Clin Aesthet Dermatol. 2011;4(8):31-49.

11. Lee T, Friedman A. Skin barrier health: regulation and repair of the stratum corneum and the role of over-the-counter skin care. J Drugs Dermatol. 2016;15(9):1047-1051.

12. Jownie JB. Understanding moisturizers and their clinical benefits. Pract Dermatol Pediatr. 2010:19-22.
13. Zeichner JA, del Rosso JQ. Multivesicular emulsion ceramidecontaining moisturizers: an evaluation of their role in the management of common skin disorders. J Clin Aesthet Dermatol. 2016;9(12): 26-32.

14. Vogel CA, Balkrishnan R, Fleischer AB, Cayce KA, Feldman SR. Over-the-counter topical skin products - a common component of skin disease management. Cutis. 2004;74(1):55-67.

15. Courage W. Hardware and measuring principle: Corneometer. In: Elsner P, Berardesca E, Maibach HI, editors. Vol. I. Bioengineering of the Skin: Water and the Stratum Corneum. Philadelphia: CRC Press; 1994:171-176.

16. Distante F, Berardesca E. Transepidermal water loss. In: Berardesca E, Elsner P, Wilhelm K-P, Maibach HI, editors. Bioengineering of the Skin: Methods and Instrumentation Volume III. Philadelphia: CRC Press; 1995.

17. Wilkinson DS, Fregert S, Magnusson B, et al. Terminology of contact dermatitis. Acta Derm Venereol. 1970;50(4):287-292.

18. Draize JH, Woodard G, Calvery HO. Methods for the study of irritation and toxicity of substances applied topically to the skin and mucous membranes. J Pharmacol Exp Ther. 1944;82(3):377-390.

19. Kraft JN, Lynde CW. Moisturizers: what they are and a practical approach to product selection. Skin Therapy Lett. 2005;10(5):1-8.

20. Lynde CW. Moisturizers: what they are and how they work. Skin Therapy Lett. 2001;6(13):3-5.

21. Rawlings AV, Davies A, Carlomusto M, et al. Effect of lactic acid isomers on keratinocyte ceramide synthesis, stratum corneum lipid levels and stratum corneum barrier function. Arch Dermatol Res. 1996;288(7):383-390.

22. Soma Y, Kashima M, Imaizumi A, Takahama H, Kawakami T, Mizoguchi M. Moisturizing effects of topical nicotinamide on atopic dry skin. Int J Dermatol. 2005;44(3):197-202.

23. Alani JI, Davis MD, Yiannias JA. Allergy to cosmetics: a literature review. Dermatitis. 2013;24(6):283-290.

24. Wiechers JW. Formulating at $\mathrm{pH} 4-5$ : How lower $\mathrm{pH}$ benefits the skin and formulations. Cosmet Toilet. 2008;123(12):61-70.

25. Imokawa G, Abe A, Jin K, Higaki Y, Kawashima M, Hidano A. Decreased level of ceramides in stratum corneum of atopic dermatitis: an etiologic factor in atopic dry skin? J Invest Dermatol. 1991;96(4):523-526.
Clinical, Cosmetic and Investigational Dermatology

\section{Publish your work in this journal}

Clinical, Cosmetic and Investigational Dermatology is an international, peer-reviewed, open access, online journal that focuses on the latest clinical and experimental research in all aspects of skin disease and cosmetic interventions. This journal is included on PubMed. The manuscript management system is completely online

\section{Dovepress}

and includes a very quick and fair peer-review system, which is all easy to use. Visit http://www.dovepress.com/testimonials.php to read real quotes from published authors 